\title{
Synergistic Interferon Alpha-based Drug Combinations Inhibit SARS-CoV-2 and other viral Infections in Vitro
}

\section{Aleksandr lanevski}

NTNU: Norges teknisk-naturvitenskapelige universitet

\section{Rouan Yao}

NTNU: Norges teknisk-naturvitenskapelige universitet

\section{Eva Zusinaite}

Tartu Ülikool: Tartu Ulikool

Laura Lello

Tartu Ülikool: Tartu Ulikool

\section{Sainan Wang}

Tartu Ülikool: Tartu Ulikool

\section{Einji Jo}

Institut Pasteur Korea

Jaewon Yang

Institut Pasteur Korea

Hilde Lysvand

NTNU: Norges teknisk-naturvitenskapelige universitet

\section{Kirsti Loseth}

NTNU Universitetsbibliotek: Norges teknisk-naturvitenskapelige universitet

\section{Valentyn Oksenych}

University of Oslo: Universitetet i Oslo

\section{Tanel Tenson}

Tartu Ülikool: Tartu Ulikool

\section{Marc Windisch}

Pasteur Institute Korea: Institut Pasteur Korea

\section{Minna Poranen}

Helsingin Yliopisto

\section{Anni Nieminen}

Helsingfors Universitet: Helsingin Yliopisto

\section{Svein Arne Nordb $\emptyset$}

NTNU: Norges teknisk-naturvitenskapelige universitet

\section{Mona Fenstad}


NTNU: Norges teknisk-naturvitenskapelige universitet

\section{Gunnveig Grodeland}

Oslo Universitetssykehus

\section{Pal Akrust}

UiO: Universitetet i Oslo

\section{Marius Troseid}

University of Oslo: Universitetet i Oslo

\section{Anu Kantele}

University of Helsinki: Helsingin Yliopisto

\section{Andres Merits}

Tartu Ülikool: Tartu Ulikool

\section{Magnar Bjoras}

NTNU: Norges teknisk-naturvitenskapelige universitet

\section{Denis E Kainov ( $\sim$ denis.kainov@ntnu.no)}

NTNU: Norges teknisk-naturvitenskapelige universitet https://orcid.org/0000-0001-7716-6955

\section{Research Article}

Keywords: antivirals, antiviral drug combinations, broad-spectrum antivirals, virus, interferon

Posted Date: February 16th, 2021

DOl: https://doi.org/10.21203/rs.3.rs-189394/v1

License: (c) (i) This work is licensed under a Creative Commons Attribution 4.0 International License. Read Full License 


\title{
Synergistic interferon alpha-based drug combinations
}

\section{inhibit SARS-CoV-2 and other viral infections in vitro}

\author{
Aleksandr Ianevski ${ }^{1}$, Rouan Yao ${ }^{1}$, Eva Zusinaite ${ }^{2}$, Laura Sandra Lello ${ }^{2}$, Sainan Wang ${ }^{2}$, Eunji Jo ${ }^{3}$, Jaewon \\ Yang ${ }^{3}$, Hilde Lysvand ${ }^{1}$, Kirsti Løseth ${ }^{1}$, Valentyn Oksenych ${ }^{1,9}$, Tanel Tenson ${ }^{2}$, Marc P. Windisch ${ }^{3}$, Minna \\ Poranen ${ }^{4}$, Anni I. Nieminen 5, Svein Arne Nordbø 1,6, Mona Høysæter Fenstad 1,7, Gunnveig Grødeland 8,9,10, \\ Pål Aukrust 8,9,10, Marius Trøseid 8,9,10, Anu Kantele 11, Andres Merits ${ }^{2}$, Magnar Bjørås 1, Denis E. Kainov 1,2,4* \\ 1 Department of Clinical and Molecular Medicine (IKOM), Norwegian University of Science and Technology, \\ 7028, Trondheim, Norway \\ 2 Institute of Technology, University of Tartu, 50411 Tartu, Estonia \\ 3 Applied Molecular Virology Laboratory, Institut Pasteur Korea, Sampyeong-dong 696, 463-400, Bundang-gu,
} Seongnam-si, Gyeonggi-do, Korea

4 Molecular and Integrative Biosciences Research Programme, Faculty of Biological and Environmental Sciences, 00014 University of Helsinki, Finland

5 Institute for Molecular Medicine Finland, FIMM, University of Helsinki, 00014, Helsinki, Finland

6 Department of Medical Microbiology, St. Olavs Hospital, 7006, Trondheim, Norway

7 Department of immunology and transfusion medicine, St. Olavs Hospital, 7006 Trondheim, Norway

8 Research Institute of Internal Medicine, Oslo University Hospital Rikshospitalet, 0372, Oslo, Norway

9 Institute of Clinical Medicine (KlinMed), University of Oslo, 0318, Oslo, Norway

10 Section of Clinical Immunology and Infectious diseases, Oslo University Hospital Rikshospitalet, 0372, Oslo, Norway

11 Inflammation Center, Infectious Diseases, Helsinki University Hospital and University of Helsinki, Meilahti Vaccine Research Center, 00029 HUS, Helsinki, Finland

*: Correspondence: denis.kainov@ntnu.no; Tel./Fax: +358-405-490-220

Running title: IFNa-based combinations

Keywords: antivirals; antiviral drug combinations; broad-spectrum antivirals; virus; interferon

\begin{abstract}
There is an urgent need for new antivirals with powerful therapeutic potential and tolerable side effects. In the present study, we found that recombinant human interferon-alpha (IFNa) triggered cell intrinsic and extrinsic antiviral responses and reduced replication of severe acute respiratory syndrome coronavirus 2 (SARS-CoV-2) in human lung epithelial Calu-3 cells. However, IFNa alone was insufficient to completely abolish SARS-CoV-2 replication. Combinations of IFNa with camostat, remdesivir, EIDD-2801, cycloheximide or convalescent serum showed strong synergy and effectively inhibited SARS-CoV-2 infection. Additionally, we demonstrated synergistic antiviral activity of IFNa2a with pimodivir against influenza A virus (FluAV) infection in human lung epithelial A549
\end{abstract}


cells, as well as of IFNa2a with lamivudine against human immunodeficiency virus 1 (HIV-1) infection in human TZM-bl cells. Our results indicate that IFNa2a-based combinational therapies help to reduce drug dose and improve efficacy in comparison with monotherapies, making them attractive targets for further pre-clinical and clinical development.

\section{Introduction}

Every year, emerging and re-emerging viruses surface from natural reservoirs to infect, disable, and kill people [1,2]. These outbreaks can be devastating to public health and have the capacity to ruin local and global economies when left unaddressed. According to the World Health Organization, there is an urgent need for better control of emerging and re-emerging viral diseases, including COVID-19.

Antiviral drugs are powerful tools to combat emerging viral diseases. Among these are recombinant human interferons (IFNs), which have been approved as a monotherapy for treatment of hepatitis $\mathrm{C}$ virus (HCV) and hepatitis B virus (HBV) infections [3]. They have also been shown to be effective against other viruses in clinical trials and in laboratory settings [4-6]. Due to their broad activity, IFNs can be considered broad-spectrum antivirals.

IFNs are a large class of host proteins that trigger an innate defense against viruses $[7,8]$. They are classified into three groups, according to the cellular receptor they bind. Type I IFNs consist of IFNalpha (IFNa), IFN-beta (IFNb), IFN-epsilon, IFN-kappa and IFN-omega (IFNw) and bind to the IFNalpha/beta receptor (IFNAR1/2). Type II IFNs consist of IFN-gamma (IFNg) and interact with IFNGR1/2. Finally, type III IFNs, consisting of IFN-lambda-1/IL29 (IFN11), IFN-lambda-2/IL28A (IFN12), IFN-lambda-3/IL28B (IFN13) and IFN-lambda-4 (IFN14), pass signals through a receptor complex consisting of interleukin (IL) IL10R2 and IFNLR1 [9].

IFNs also induce transcription of interferon-stimulated genes (ISGs), which participate in intrinsic antiviral and extrinsic immune responses. For example, ISGs, such as IFIT1 and OASL, activate Ribonuclease L (RNaseL), which leads to the degradation of viral RNA [10]. ISGs such as interleukins (ILs), C-X-C and C-C motif chemokines (CXCLs and CCLs) recruit immune cells to the site of infection. Notably, mutations in IFN-signaling pathway genes have resulted in increased susceptibility to viral infections and reduced survival of patients [11-14].

However, recombinant IFNs possess limited antiviral efficacy and can cause adverse effects when used as monotherapies [15]. For example IFNb has been used on hospitalized patients with COVID-19 with no or limited efficacy, as indicated by overall mortality, initiation of ventilation, and duration of hospital stay $[16,17]$.

Synergistic combinations of IFNs with other antiviral drugs can increase effectiveness and overcome toxicities by countering biological compensation, allowing for reduced doses of each 
compound to be used (Fig. S1). For example, combination treatment of IFNa and ribavirin has been the "gold standard" for treatment of chronic HCV infection for more than decade. Additionally, a combination of IFNb1b, lopinavir-ritonavir, and ribavirin has been tested for treatment of hospitalized patients with COVID-19 in an open-label, randomized, phase II trial [18]. Combined use of IFNa2b and IFNg has also been evaluated in patients positive to SARS-CoV-2 [19]. Finally, combined use of IFNa and arbidol has been tested in a cohort of confirmed COVID-19 cases in Wuhan, China [20]. These treatments were shown to positively impact the resolution of the COVID-19 symptoms.

Here, we identified several novel synergistic IFNa2a-based drug combinations against SARS-CoV2, FluAV and HIV-1. These treatment combinations were effective at lower concentrations compared to IFNa2a monotherapies. We also propose the need for further investigation due to their powerful treatment potential, which can be leveraged for use in response to imminent viral threats including the emergence and re-emergence of viruses, immune-evading or drug-resistant variants, and viral coinfections.

\section{Results}

Type I IFNs reduce SARS-CoV-2 replication in vitro more efficiently than type II and III IFNs

Although dexamethasone has been shown to improve survival of patients with severe or critical COVID-19 [21], there are currently no curative therapies against SARS-CoV-2. However, previous studies have uncovered several potent antiviral agents including IFNs against SARS-CoV-2 in vitro and in vivo $[4,5,22]$. Here, we tested type I, II, and III IFNs against wild type SARS-CoV-2 in Calu-3 and Vero-E6 cells using cell viability and virus plaque reduction assays as readouts. We observed that type I IFNs rescued both cell types from virus-mediated death and reduced SARS-CoV-2 replication more efficiently than type II and III IFNs. However, the rescue was only partial, and virus replication was reduced only by 2-3-folds (Fig. 1).

Type I IFNs reduce the SARS-CoV-2 RNA replication and accelerate virus-mediated induction of cellular type III IFNs, IFNbI and ISGs

To shed more light on the mechanism of action of type I IFNs, we evaluated their effect on expression of cellular genes and transcription of viral RNA in mock- and SARS-CoV-2-infected Calu-3 cells. For this, cells were treated with $1 \mu \mathrm{g} / \mathrm{mL}$ of type I IFNs or vehicle and infected with virus or mock. After $24 \mathrm{~h}$, we analyzed polyadenylated RNA using RNA-sequencing. We found that type I IFNs attenuated production of viral RNA (Fig. 2a), while increasing expression of many ISGs in both mockand virus-infected cells (Fig. 2b). These include IFIT1, IFIT2 and IFIT3, which play a role in recognition of viral RNA; OASL and OAS2, which are involved in RNase L-mediated RNA degradation; and IDO1 
which is essential for kynurenine biosynthesis [23-26]. Interestingly, type I IFNs boosted virus-activated expression of type III IFNs (IFN11, IFN12, IFN13 and IFN14) as well as IFNb1, which belongs to type I IFN. These results indicate that type I IFNs not only trigger expression of ISGs regardless of infection, but also amplify expression of other interferons usually activated by viral infections.

Next, we studied the effect of type I IFNs on the metabolism of mock- and SARS-CoV-2 -infected Calu-3 cells. We analyzed mainly polar metabolites in cell cultures at 24 hpi (Fig. S2; Fig. S2). A total of 93 metabolites were quantified. Viral infection substantially lowered tyrosine and 4-hydroxyproline levels $\left(\log _{2} \mathrm{FC}<-2\right)$. Regardless of viral infection, administration of type I IFNs lowered the levels of several metabolites including tryptophan while increasing kynurenine $\left(\log _{2} \mathrm{FC}>3\right.$; Fig. 2c). This indicates that type I IFNs activate IDO1-mediated kynurenine biosynthesis, which is responsible for adverse reactions such as suppression of T-cell responses, pain hypersensitivity and behavior disturbance [27].

IFNa1b, IFNa2a and IFNw1 are more effective than IFNb1a against SARS-CoV-2 in Calu-3 cells

To identify the type I IFN with most activity against SARS-CoV-2 infection, we infected IFNtreated and IFN-untreated Calu-3 cells with SARS-CoV-2-mCherry (moi 0.01) and collected media from the cells (P1) after $48 \mathrm{~h}$. The media were diluted 25-fold and applied to noninfected cells for another $48 \mathrm{~h}$ (P2). Mock-infected cells were used as controls. The experiment is schematically depicted in Fig. 3a. Fluorescence microscopy, fluorescence intensity analysis, and cell viability assay of P1 and P2 cells showed that IFNa1b, IFNa2a and IFNw1 were more effective inhibitors of SARS-CoV-2 infection than IFNb1a. However, none of the IFNs tested able to inhibit virus infection completely (Fig. $3 b-d)$.

Anti-SARS-CoV-2 activity of IFNa2a depends on virus load and time of addition

IFNas are widely used in basic research to elucidate biological activities, structure and mechanism of action of type I IFNs. They are encoded by multiple genes and vary slightly from one another in their protein structure. We tested IFNa2a against various doses of SARS-CoV-2-mCherry in Calu-3 cells. Calu-3 cells were treated with $1 \mu \mathrm{g} / \mathrm{mL}$ IFNa2a, then infected with SARS-CoV-2-mCherry at the indicated moi. After $48 \mathrm{~h}$, fluorescent intensity and cell viability analysis were performed. We found that efficacy of IFNa2a treatment in preventing SARS-CoV-2 infection was dependent on virus load, decreasing in efficacy as moi increases (Fig. 4a).

We also tested whether time of IFNa2a addition could influence its anti-SARS-CoV-2 activity. To this end, we treated Calu-3 cells with $1 \mu \mathrm{g} / \mathrm{mL}$ IFNa2a at indicated time points and infected the cells with SARS-CoV-2-mCherry $(\mathrm{moi}=0.01)$. After $48 \mathrm{~h}$ of infection, fluorescent intensity and cell viability 
analysis were performed. We found that efficacy of IFNa2a treatment was dependent on time of administration, showing more efficacy when given prior virus infection than following infection (Fig. $4 b)$.

Synergistic IFNa2a-based Combinations Against SARS-CoV-2 infection

Next, we examined whether combinations of IFNa2a with several known inhibitors of SARS-CoV2 infection can protect cells from virus infection more efficiently than IFNa2a alone. For this, we first confirmed antiviral activities of remdesivir, EIDD-2801, camostat, cycloheximide, and convalescent serum [28-32] on Calu-3 cells using SARS-CoV-2-mCherry (Fig. S3). Then, we tested the antiviral efficacy of these agents in combination with IFNa2a in Calu-3 cells by monitoring virus-mediated mCherry expression and cell viability. Each drug combination was tested in a $6 \times 6$ dose-response matrix, where 5 doses of single drugs are combined in a pairwise manner. As a result, we obtained doseresponse matrices demonstrating virus inhibition and cell viability achieved by each combination (Fig 5a,b; Fig. S4). We plotted synergy distribution maps, showing synergy at each pairwise dose. For each drug combination, we calculated ZIP synergy scores and most synergistic area scores (Fig. 5c). We observed that all combinations were synergistic based on fluorescent intensity or cell viability analyses (synergy scores $>10$ ). However, camostat-IFNa2a and convalescent plasma-IFNa2a were the most active in terms of both dose-response effect and synergism. This high synergy allowed us to substantially decrease the concentration of both components (especially IFNa2a) to achieve antiviral efficacy that was comparable to those of individual drugs at high concentrations.

Based on our eperiments, we propose the following mechanism of action of the IFNa combinations (Fig. 6d). IFNa induces transcription of ISGs including IFIT1, IFIT2 and IFIT3, which recognize viral RNA; OASL and OAS2, which are involved in RNase L-mediated RNA degradation; and IDO1, which catalyzes kynurenine biosynthesis. IFNa2a also facilitates expression of several cytokines and virusactivated synthesis of IFNL1, IFNL2, IFNL3, IFNL4, and IFNB1, which alarm the neighboring cells of upcoming infection. Convalescent serum contains neutralizing antibodies which bind S protein of SARS-CoV-2 preventing virus entry into the cells [16]. Camostat, a serine protease inhibitor, reduces SARS-CoV-2-cell membrane fusion by binding host TMPRSS2 [33]. In addition, camostat possesses some potential beneficial immunomodulatory effects by interfering with the bradykinin/kallikrein pathways [34]. Remdesivir and EIDD-2801 are nucleoside analogues which inhibit viral RNA synthesis [30, 35]. Cycloheximide inhibits translation elongation and, thereby, reduces SARS-CoV-2 replication [29]. Therefore, combinations of IFNa2a with therapies targeting viral entry or replication inhibit infection within a virus-host system. These combinations lowered toxicity and improved efficacy in 
comparison with monotherapies in vitro, making them attractive targets for further pre-clinical and clinical development.

Novel IFNa2a-based Combinations Against FluAV Infections

In order to extend our findings beyond SARS-CoV-2, we tested IFNa2a in combinations with known influenza inhibitors ofemetine, flavopiridol, camostat, obatoclax, SNS-032, gemcitabine, monensin, cycloheximide or pimodivir [10,36,37] against FluAV infection in A549 cells. Cell viability was measured after $48 \mathrm{~h}$ in FluAV- and mock-infected cells to determine efficiency and toxicity of each compound and their combinations with IFNa2a (Fig. 6). We observed that combination of IFNa2a and pimodivir was synergistic against FluAV infection (ZIP synergy score: 22.13, the most synergistic area score: 42.77) while remaining nontoxic at synergistic doses for either drug. Given that pimodivir (VX787, JNJ-63623872) is an orally available anti-FluAV agent which inhibits cap-snatching domain of viral polymerase basic protein 2 [38] and has shown promising results in Phase II clinical trials [39], we believe that its combination with IFNa warrants further preclinical and clinical investigations.

Novel IFNa2a-based Combinations Against HIV-1 Infections

We also tested IFNa2a in combination with known anti-retrovirals agents, lamivudine, brequinar, tenofovir, suramin, clofarabine and decitabine [40-43] against HIV-1 in TZM-bl cells. Cell viability and HIV-induced luciferase expression were measured for each compound or their combination with IFNa2a after $48 \mathrm{~h}$. We identified that IFNa2a and lamivudine in combination was most effective while being nontoxic at synergistic drug concentrations, with ZIP synergy scores of 5.7 and ZIP synergy score of 11.4 at the most synergistic area (Fig. 7). Given that lamivudine (3TC) is an orally available anti-HIV drug which inhibits viral reverse transcriptase [44], we believe that its combination with IFNa also warrants further preclinical and clinical investigations.

\section{Discussion}

Monotherapies are mainly used to treat emerging viral infections due to lack of studies on drugdrug interactions [45]. Here, we have reported several novel synergistic IFNa-based combinations that have better efficacy than single drug therapies. In particular, we report novel anti-SARS-CoV-2 activities of IFNa2a in combination with remdesivir, EIDD-2801, camostat, cycloheximide, and convalescent serum in vitro. The capacity to deliver these combinations through different routes could provide a path for effective treatment of COVID-19 patients at different stages of diseases [33, 46, 47]. Thus, combination of these drugs with IFNa2a could be of relevance for patients, including patients with immune suppression including lymphopenia. 
We also identified synergistic activity in the combined administration of IFNa2a with the investigational orally available pimodivir against FluAV, as well as of IFNa2a and approved orally available lamivudine against HIV-1 infection. These combinations may provide a relatively convenient treatment option that can be administered outside of a hospital setting.

Interestingly, pimodivir, lamivudine, remdesivir, and EIDD-2801 interfere with synthesis of viral nucleic acids but not with the development of innate immune response [30, 35, 38, 48]. This also supports the clinical use of IFNa-remdesivir (or EIDD-2801), IFNa-pimodivir and IFNa-lamivudine combinations as a treatment for SARS-CoV-2, FluAV and HIV-1 infections, respectively.

Thus, we have identified combination treatments that reduce viral replication in vitro at lower concentrations than is required with monotherapies. The low effective doses of combination drugs may have several clinical advantages, including improved patient outcome and fewer adverse events associated with IFN-mediated hypercytokinemia, suppression of T-cell response, pain hypersensitivity, and behavior disturbance [23]. We believe further development of these antiviral combinations can lead to practical treatment options that are more effective while having potentially reduced side effects than currently existing treatments.

\section{Experimental procedures}

Drugs, cells and viruses

Table S1 lists IFNs and other antiviral agents, their suppliers and catalogue numbers. Lyophilized IFNs were dissolved in sterile deionized water to obtain $200 \mu \mathrm{g} / \mathrm{mL}$ concentrations. To obtain $10 \mathrm{mM}$ stock solutions, compounds were dissolved in dimethyl sulfoxide (DMSO; Sigma-Aldrich, Hamburg, Germany) or milli-Q water. The convalescent serum (G614) obtained from a recovered COVID-19 patient has been described in a previous study [22].

The propagation of human non-small cell lung cancer Calu-3, human adenocarcinoma alveolar basal epithelial A549, African green monkey kidney Vero-E6, T-cell like ACH-2 cells, which possess a single integrated copy of the provirus HIV-1 strain LAI (NIH AIDS Reagent Program), and human cervical cancer derived TZM-bl, which express firefly luciferase under control of HIV-1 long terminal repeat (LTR) promoter allowing quantitation of the viral infection (tat-protein expression by integrated HIV-1 provirus) using firefly luciferase assay, have been described in our previous studies [22, 40-43].

The propagation of wild-type SARS-CoV-2 (hCoV-19/Norway/Trondheim-S15/2020), recombinant mCherry-expressing SARS-CoV-2 strains (SARS-CoV-2-mCherry), wild type human influenza A/Udorn/307/1972 (H3N2) and HIV-1 have been also described previously [22, 40-43](Rihn et al., PBIOLOGY-D-20-02646R2). To quantitate the production of infectious virions, we titered the viruses using plaque assays or ELISA [22, 40-43]. 


\section{Drug Testing and Drug Sensitivity Quantification}

Approximately $4 \times 10^{4}$ Vero-E6 or Calu-3 cells were seeded per well in 96-well plates. The cells were grown for $24 \mathrm{~h}$ in DMEM or DMEM-F12, respectively, supplemented with 10\% FBS and Pen-Strep. The medium was then replaced with DMEM or DMEM-F12 containing 0.2\% BSA, Pen-Strep and the compounds in 3-fold dilutions at 7 different concentrations. No compounds were added to the control wells. The cells were uninfected (mock) or infected with SARS-CoV-2 or SARS-CoV-2-mCherry strains at a multiplicity of infection (moi) of 0.01 . After $72 \mathrm{~h}$ of infection, a CellTiter-Glo (CTG) assay was performed to measure cell viability. Drug efficacy on SARS-CoV-2-mCherry infected cells was measured on PFA- or acetone-fixed cells with fluorescence.

For testing compound toxicity and efficacy against FluAV, approximately $4 \times 10^{4} \mathrm{~A} 549$ cells were seeded in each well of a 96-well plate. The cells were grown for $24 \mathrm{~h}$ in growth medium in DMEM supplemented with 10\% FBS and Pen-Strep. The medium was then replaced with DMEM containing $0.2 \%$ BSA, Pen-Strep, $0,5 \mu \mathrm{g} / \mathrm{mL}$ TPSK-trypsin and compounds in three-fold dilutions at seven different concentrations. No compounds were added to the control wells. The cells were infected with FluAV (moi $=0.5$ ) or mock. At $48 \mathrm{hpi}$, the media was removed, and a CTG assay was performed to measure cell viability.

For testing compound toxicity and efficacy against HIV-1, approximately $4 \times 10^{4} \mathrm{TZM}-\mathrm{bl}$ cells were seeded in each well of a 96-well plate in growth medium in DMEM supplemented with 10\% FBS and Pen-Strep. The cells were grown for $24 \mathrm{~h}$ in growth medium. The medium was then replaced with DMEM containing $0.2 \%$ BSA, Pen-Strep and the compounds in 3-fold dilutions at 7 different concentrations. No compounds were added to the control wells. The cells were infected with HIV-1 (corresponding to $300 \mathrm{ng} / \mathrm{mL}$ of HIV-1 p24) or mock. At 48 hours post-infection (hpi), the media was removed from the cells, the cells were lysed, and firefly luciferase activity was measured using the Luciferase Assay System (Promega, Madison, WI, USA). In a parallel experiment, a CTG assay was performed to measure cell viability.

The half-maximal cytotoxic concentration $\left(\mathrm{CC}_{50}\right)$ for each compound was calculated based on viability/death curves obtained on mock-infected cells after non-linear regression analysis with a variable slope using GraphPad Prism software version 7.0a. The half-maximal effective concentrations (EC50) were calculated based on the analysis of the viability of infected cells by fitting drug doseresponse curves using four-parameter $(4 P L)$ logistic function $f(x)$ :

$$
f(x)=A_{\min }+\frac{A_{\max }-A_{\min }}{1+\left(\frac{x}{m}\right)^{\lambda}},
$$

where $f(x)$ is a response value at dose $x, A_{\min }$ and $A_{\max }$ are the upper and lower asymptotes (minimal and maximal drug effects), $m$ is the dose that produces the half-maximal effect (EC50 or $\mathrm{CC}_{50}$ ), and $\lambda$ is 
the steepness (slope) of the curve. The relative effectiveness of the drug was defined as selectivity index $\left(S I=C_{50} / E_{50}\right)$.

To quantify each drug responses in a single metric, a drug sensitivity score (DSS) was calculated as a normalized version of standard area under dose-response curve $(A U C)$, with the baseline noise subtracted, and normalized maximal response at the highest concentration (often corresponding to off-target toxicity):

$$
D S S=\frac{A U C-t\left(x_{\max }-x_{\min }\right)}{(100-t)\left(x_{\max }-x_{\min }\right) \log _{10} A \min ^{\prime}},
$$

where activity threshold $t$ equals $10 \%$, and DSS $\in[0,50]$.

\section{Drug Combination Test and Synergy Calculations}

Calu-3, A549 or TZM-bl cells were treated with different concentrations of two drugs and infected with SARS-CoV-2-mCherry (moi = 0.01), FluAV (moi = 0.5), HIV-1 (corresponding to $300 \mathrm{ng} / \mathrm{mL}$ of HIV1 p24) or mock. After $48 \mathrm{~h}$, cell viability and reporter protein expression (SARS-CoV-2-mCherry and HIV-1) were measured. To test whether the drug combinations act synergistically, the observed responses were compared with expected combination responses. The expected responses were calculated based on the ZIP reference model using SynergyFinder version 2 [49, 50]. Final synergy scores were quantified as average excess response due to drug interactions (i.e., 10\% of cell survival beyond the expected additivity between single drugs represents a synergy score of 10). Additionally, for each drug combination, we report a most synergistic area score - the most synergistic 3-by-3 dosewindow in a dose-response matrix.

\section{Gene Expression Analysis}

Calu-3 cells were treated with $1 \mu \mathrm{g} / \mathrm{ml}$ IFNa2a or vehicle control. Cells were infected with SARSCoV-2-mCherry at a moi of 0.01 or mock. After $24 \mathrm{~h}$, total RNA was isolated using RNeasy Plus Mini kit (Qiagen, Hilden, Germany). Polyadenylated mRNA was isolated from 250 ng of total RNA with NEBNext Poly(A) mRNA magnetic isolation module. NEBNext Ultra II Directional RNA Library Prep kit from Illumina was used to prepare samples for sequencing. Sequencing was done on NextSeq 500 instrument (set up: single-end $1 \times 76 \mathrm{bp}+$ dual index $8 \mathrm{bp}$ ) using NextSeq High Output 75 cycle sequencing kit (up to $400 \mathrm{M}$ reads per flow cell). Reads were aligned using the Bowtie 2 software package version 2.4.2 to the NCBI reference sequence for SARS-CoV-2 (NC_045512.2) and to the human GRCh38 genome. The number of mapped and unmapped reads that aligned to each gene were obtained with the featureCounts function from Rsubread R-package version 2.10. The GTF table for the reference $\begin{array}{llll}\text { sequence } & \text { was } & \text { downloaded }\end{array}$ https://ftp.ncbi.nlm.nih.gov/genomes/all/GCF/009/858/895/GCF_009858895.2_SM985889v3/GCF_00985 
8895.2_ASM985889v3_genomic.gtf.gz. The heatmaps were generated using the pheatmap package (https://cran.r-project.org/web/packages/pheatmap/index.html) based on log2-transformed profiling data.

\section{Metabolic Analysis}

Calu-3 cells were treated with $1 \mu \mathrm{g} / \mathrm{ml}$ IFNa2a or vehicle control. Cells were infected with SARSCoV-2-mCherry at a moi 0.01 or mock. After $24 \mathrm{~h}$, virus was inactivated with UVC radiation and trypsin treatment as described previously [22].

Metabolites were extracted from $100 \mu \mathrm{l}$ of cell culture medium with $400 \mu \mathrm{L}$ of cold extraction solvent (acetonitrile:methanol:water; 40:40:20). Subsequently, samples were sonicated for 3 cycle (60 s, power $=60$ and frequency $=37$ ), vortexed for $2 \mathrm{~min}$ and centrifuged at $4{ }^{\circ} \mathrm{C}, 14000 \mathrm{rpm}$ for $10 \mathrm{~min}$. The supernatant was transferred to autosampler vials for LC-MS analysis. The extracts were analyzed with Thermo Vanquish UHPLC+ system coupled to a QExactive Orbitrap quadrupole mass spectrometer equipped with a heated electrospray ionization (H-ESI) source probe (Thermo Fischer Scientific, Waltham, MA, USA). A SeQuant ZIC-pHILIC $(2.1 \times 100 \mathrm{~mm}, 5 \mu \mathrm{m}$ particles $)$ HILIC phase analytical column (Merck KGaA, Darmstadt, Germany) was used as a chromatographic separation column.

Gradient elution was carried out with a flow rate of $0.100 \mathrm{~mL} / \mathrm{min}$ with $20 \mathrm{mM}$ ammonium carbonate, adjusted to $\mathrm{pH} 9.4$ with ammonium solution (25\%) as mobile phase A and acetonitrile as mobile phase B. The gradient elution was initiated from $20 \%$ mobile phase A and $80 \%$ mobile phase B and maintained for $2 \mathrm{~min}$. Then, mobile phase A was gradually increased up to $80 \%$ for $17 \mathrm{~min}$, followed by a decrease to $20 \%$ over the course of $17.1 \mathrm{~min}$. and sustained for up to $24 \mathrm{~min}$.

The column oven and auto-sampler temperatures were set to $40 \pm 3^{\circ} \mathrm{C}$ and $5 \pm 3{ }^{\circ} \mathrm{C}$, respectively. The mass spectrometer was equipped with a heated electrospray ionization (H-ESI) source using polarity switching and the following settings: resolution of 35,000, the spray voltages of $4250 \mathrm{~V}$ for positive and $3250 \mathrm{~V}$ for negative mode, sheath gas at 25 arbitrary units (AU), the auxiliary gas at $15 \mathrm{AU}$, sweep gas flow of 0, Capillary temperature of $275^{\circ} \mathrm{C}$, and S-lens RF level of 50.0. Instrument control was operated with Xcalibur 4.1.31.9 software (Thermo Fischer Scientific, Waltham, MA, USA). Metabolite peaks were confirmed using the mass spectrometry metabolite library kit MSMLS-1EA (Sigma Aldrich supplied by IROA Technologies).

For data processing, final peak integration was done with the TraceFinder 4.1 software (Thermo Fisher Scientific, Waltham, MA, USA) and for further data analysis, the peak area data was exported as an Excel file. Data quality was monitored throughout the run using pooled healthy human serum as Quality Control (QC), which was processed and extracted in the same manner as unknown samples. 
After integration of QC data with TraceFinder 4.1, each detected metabolite was checked and \%RSD were calculated, while the acceptance limit was set to $\leq 20 \%$.

Blank samples were injected after every five runs to monitor any metabolite carryover. A carryover limit of $\leq 20 \%$ was set for each metabolite. Percentage background noise was calculated by injecting a blank sample at the beginning of the run. The acceptance limit for background noise was set at $\leq 20 \%$ for each metabolite.

Ethics approval and consent to participate: Standard operational procedures were approved by institutional safety committee.

Consent for publication: All authors have read and agreed to the published version of the manuscript. Availability of data and material: All data generated or analysed during this study are included in this published article and its supplementary information files

Competing interests: Authors declare no competing interests.

Author Contributions: All authors contributed to the methodology, software, validation, formal analysis, investigation, resources, data curation, writing, and review and editing of the manuscript. D.K. conceptualized, supervised, and administrated the study and acquired funding.

Funding: This research was funded by the European Regional Development Fund, the Mobilitas Pluss Project MOBTT39 (to D.K.). This work was financially supported by a National Research Foundation of Korea (NRF) grant funded by the Korean government (MSIT) (NRF-2017M3A9G6068246 and 2020R1A2C2009529). FIMM metabolomics unit was supported by HiLIFE and Biocenter Finland.

Acknowledgments: We thank personnel of Biomedicum function genomics (FuGu), Juho Vaananen and Martyn unit for transcriptomics analysis.

Conflicts of Interest: The authors declare no conflicts of interest.

\section{References}

1. Vos, T., et al., Global, regional, and national incidence, prevalence, and years lived with disability for 328 diseases and injuries for 195 countries, 1990\&\#x2013;2016: a systematic analysis for the Global Burden of Disease Study 2016. The Lancet, 2017. 390(10100): p. 1211-1259.

2. Hay, S.I., et al., Global, regional, and national disability-adjusted life-years (DALYs) for 333 diseases and injuries and healthy life expectancy (HALE) for 195 countries and territories, 1990-2016: a systematic analysis for the Global Burden of Disease Study 2016. The Lancet, 2017. 390(10100): p. 1260-1344.

3. $\quad$ Fried, M.W., et al., Peginterferon alfa-2a plus ribavirin for chronic hepatitis $C$ virus infection. N Engl J Med, 2002. 347(13): p. 975-82.

4. Lazear, H.M., J.W. Schoggins, and M.S. Diamond, Shared and Distinct Functions of Type I and Type III Interferons. Immunity, 2019. 50(4): p. 907-923.

5. Sallard, E., et al., Type 1 interferons as a potential treatment against COVID-19. Antiviral Res, 2020. 178: p. 104791. 
6. Sa Ribero, M., et al., Interplay between SARS-CoV-2 and the type I interferon response. PLoS Pathog, 2020. 16(7): p. e1008737.

7. Park, A. and A. Iwasaki, Type I and Type III Interferons - Induction, Signaling, Evasion, and Application to Combat COVID-19. Cell Host Microbe, 2020. 27(6): p. 870-878.

8. $\quad$ Felgenhauer, U., et al., Inhibition of SARS-CoV-2 by type I and type III interferons. $\mathrm{J}$ Biol Chem, 2020. 295(41): p. 13958-13964.

9. Mesev, E.V., R.A. LeDesma, and A. Ploss, Decoding type I and III interferon signalling during viral infection. Nat Microbiol, 2019. 4(6): p. 914-924.

10. Shim, J.M., et al., Influenza Virus Infection, Interferon Response, Viral CounterResponse, and Apoptosis. Viruses, 2017. 9(8).

11. Zhang, Q., et al., Inborn errors of type I IFN immunity in patients with lifethreatening COVID-19. Science, 2020. 370(6515).

12. Hadjadj, J., et al., Impaired type I interferon activity and inflammatory responses in severe COVID-19 patients. Science, 2020. 369(6504): p. 718-724.

13. Sancho-Shimizu, V., et al., Inborn errors of anti-viral interferon immunity in humans. Curr Opin Virol, 2011. 1(6): p. 487-96.

14. Pairo-Castineira, E., et al., Genetic mechanisms of critical illness in Covid-19. Nature, 2020.

15. Sleijfer, S., et al., Side effects of interferon-alpha therapy. Pharm World Sci, 2005. 27(6): p. 423-31.

16. Pan, H., et al., Repurposed Antiviral Drugs for Covid-19 - Interim WHO Solidarity Trial Results. N Engl J Med, 2020.

17. Monk, P.D., et al., Safety and efficacy of inhaled nebulised interferon beta-1a (SNG001) for treatment of SARS-CoV-2 infection: a randomised, double-blind, placebo-controlled, phase 2 trial. Lancet Respir Med, 2020.

18. Hung, I.F., et al., Triple combination of interferon beta-1b, lopinavir-ritonavir, and ribavirin in the treatment of patients admitted to hospital with COVID-19: an openlabel, randomised, phase 2 trial. Lancet, 2020. 395(10238): p. 1695-1704.

19. Idelsis, E.-M., et al., Effect and safety of combination of interferon alpha-2b and gamma or interferon alpha-2b for negativization of SARS-CoV-2 viral RNA.

Preliminary results of a randomized controlled clinical trial. medRxiv, 2020: p. 2020.07.29.20164251.

20. Zhou, Q., et al., Interferon- $\alpha 2 b$ Treatment for COVID-19. Front Immunol, 2020. 11: p. 1061.

21. Horby, P., et al., Dexamethasone in Hospitalized Patients with Covid-19 Preliminary Report. N Engl J Med, 2020.

22. Ianevski, A., et al., Potential Antiviral Options against SARS-CoV-2 Infection. Viruses, 2020. 12(6): p. 642.

23. Gaelings, L., et al., Regulation of kynurenine biosynthesis during influenza virus infection. Febs j, 2017. 284(2): p. 222-236.

24. Pichlmair, A., et al., IFIT1 is an antiviral protein that recognizes 5'-triphosphate RNA. Nat Immunol, 2011. 12(7): p. 624-30.

25. Zhou, X., et al., Interferon induced IFIT family genes in host antiviral defense. Int $\mathbf{J}$ Biol Sci, 2013. 9(2): p. 200-8.

26. Gusho, E., D. Baskar, and S. Banerjee, New advances in our understanding of the "unique" RNase L in host pathogen interaction and immune signaling. Cytokine, 2020. 133: p. 153847.

27. Boergeling, Y. and S. Ludwig, Targeting a metabolic pathway to fight the flu. Febs j, 2017. 284(2): p. 218-221. 
28. Ritchie, M.E., et al., limma powers differential expression analyses for RNAsequencing and microarray studies. Nucleic Acids Res, 2015. 43(7): p. e47.

29. Bojkova, D., et al., Proteomics of SARS-CoV-2-infected host cells reveals therapy targets. Nature, 2020. 583(7816): p. 469-472.

30. Sheahan, T.P., et al., An orally bioavailable broad-spectrum antiviral inhibits SARSCoV-2 in human airway epithelial cell cultures and multiple coronaviruses in mice. Sci Transl Med, 2020. 12(541).

31. Zhang, C.H., et al., Antiviral activity of cepharanthine against severe acute respiratory syndrome coronavirus in vitro. Chin Med J (Engl), 2005. 118(6): p. 4936.

32. Ko, M., et al., Screening of FDA-approved drugs using a MERS-CoV clinical isolate from South Korea identifies potential therapeutic options for COVID-19. bioRxiv, 2020: p. 2020.02.25.965582.

33. Breining, P., et al., Camostat mesylate against SARS-CoV-2 and COVID-19Rationale, dosing and safety. Basic Clin Pharmacol Toxicol, 2020.

34. Schmaier, A.H., The contact activation and kallikrein/kinin systems: pathophysiologic and physiologic activities. J Thromb Haemost, 2016. 14(1): p. 28-39.

35. Ko, W.C., et al., Arguments in favour of remdesivir for treating SARS-CoV-2 infections. Int J Antimicrob Agents, 2020. 55(4): p. 105933.

36. Söderholm, S., et al., Multi-Omics Studies towards Novel Modulators of Influenza A Virus-Host Interaction. Viruses, 2016. 8(10).

37. Müller, K.H., et al., Emerging cellular targets for influenza antiviral agents. Trends Pharmacol Sci, 2012. 33(2): p. 89-99.

38. $\mathrm{Fu}, \mathrm{Y}$., et al., JNJ872 inhibits influenza A virus replication without altering cellular antiviral responses. Antiviral Res, 2016. 133: p. 23-31.

39. Finberg, R.W., et al., Phase 2b Study of Pimodivir (JNJ-63623872) as Monotherapy or in Combination With Oseltamivir for Treatment of Acute Uncomplicated Seasonal Influenza A: TOPAZ Trial. J Infect Dis, 2019. 219(7): p. 1026-1034.

40. Andersen, P.I., et al., Discovery and development of safe-in-man broad-spectrum antiviral agents. Int J Infect Dis, 2020. 93: p. 268-276.

41. Andersen, P.I., et al., Novel antiviral activities of obatoclax, emetine, niclosamide, brequinar, and homoharringtonine. Viruses, 2019. 11(10): p. 964.

42. Ianevski, A., et al., Expanding the activity spectrum of antiviral agents. Drug Discov Today, 2019. 24(5): p. 1224-1228.

43. Ianevski, A., et al., Identification and Tracking of Antiviral Drug Combinations. Viruses, 2020. 12(10).

44. Perry, C.M. and D. Faulds, Lamivudine. A review of its antiviral activity, pharmacokinetic properties and therapeutic efficacy in the management of HIV infection. Drugs, 1997. 53(4): p. 657-80.

45. Antiviral Combination Database. 2020 [cited 2020 Dec 31, 2020]; Available from: http://antiviralcombi.info/.

46. Rowe, S.M., et al., Reduced sodium transport with nasal administration of the prostasin inhibitor camostat in subjects with cystic fibrosis. Chest, 2013. 144(1): p. 200-207.

47. Van Hoeven, N., et al., Pathogenesis of 1918 pandemic and H5N1 influenza virus infections in a guinea pig model: antiviral potential of exogenous alpha interferon to reduce virus shedding. J Virol, 2009. 83(7): p. 2851-61.

48. Zusinaite, E., et al., A Systems Approach to Study Immuno- and Neuro-Modulatory Properties of Antiviral Agents. Viruses, 2018. 10(8). 
49. Ianevski, A., et al., SynergyFinder: a web application for analyzing drug combination dose-response matrix data. Bioinformatics, 2017. 33(15): p. 2413-2415.

50. Ianevski, A., A.K. Giri, and T. Aittokallio, SynergyFinder 2.0: visual analytics of multi-drug combination synergies. Nucleic Acids Res, 2020. 48(W1): p. W488-w493. 


\section{Figures}

a

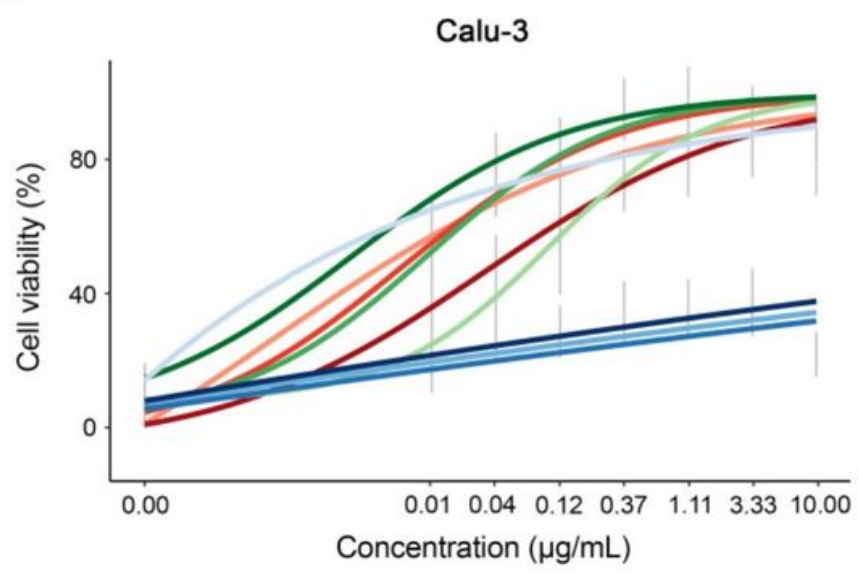

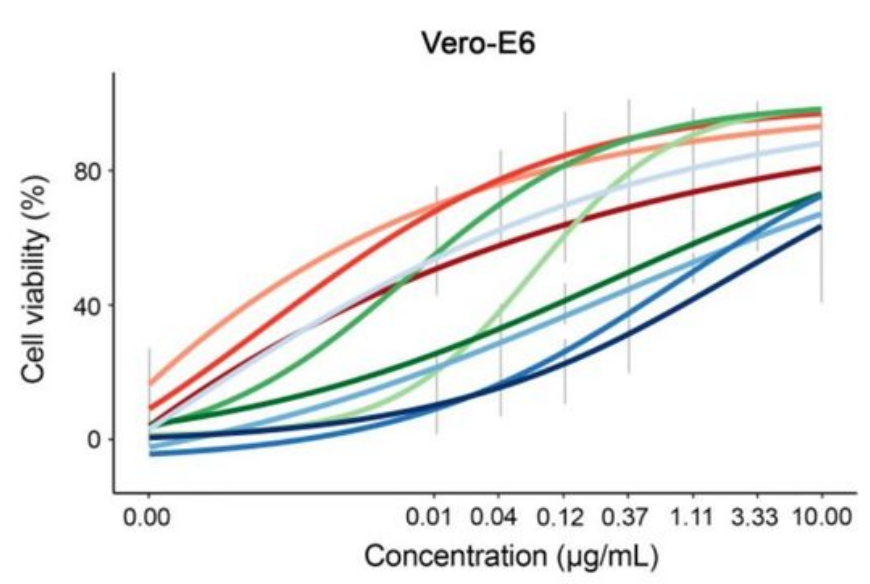

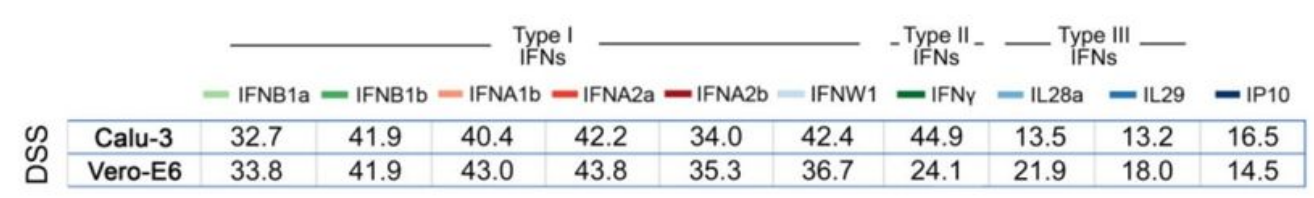

b
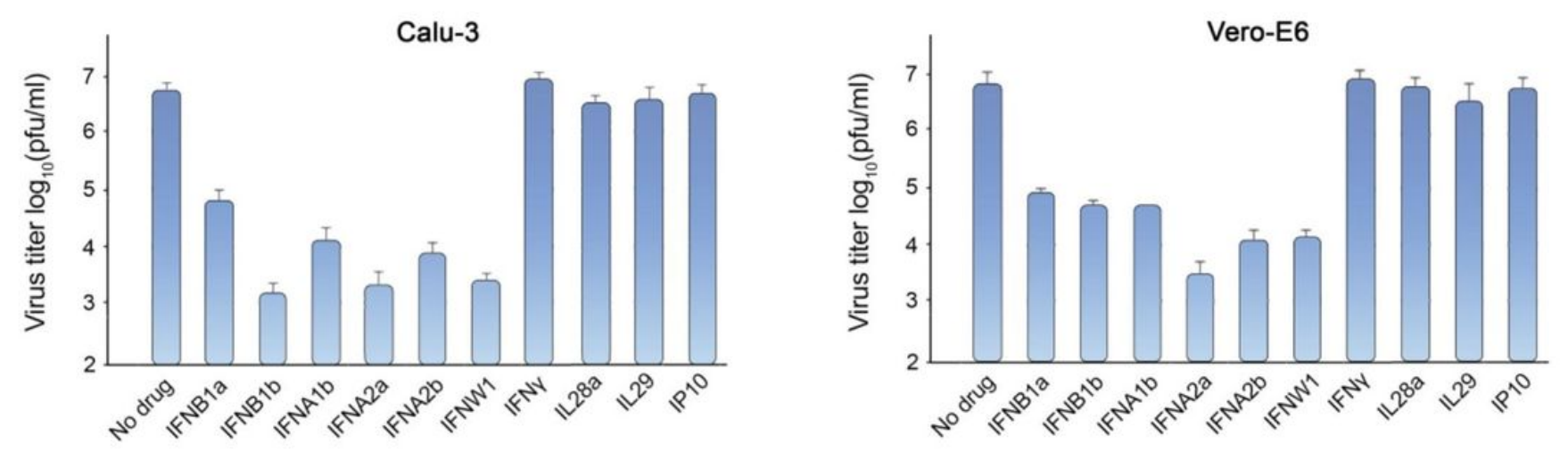

\section{Figure 1}

Type I IFNs rescue Calu-3 and Vero-E6 cells from SARS-CoV-2-mediated death and attenuate virus replication. (a) The effect of different doses of IFNs on viability of SARS-CoV-2-infected (moi $=0.01$ ) Calu3 and Vero E6 cells. Cell viability was determined using the CTG assay at 72 hpi. Mean \pm SD; $n=3$. The anti-SARS-CoV-2 activity of the IFNs was quantified using drug sensitivity scores (DSS). (b) The effects of IFNs on viral replication, measured by plaque reduction assay. Mean $\pm S D ; n=3$. 
a

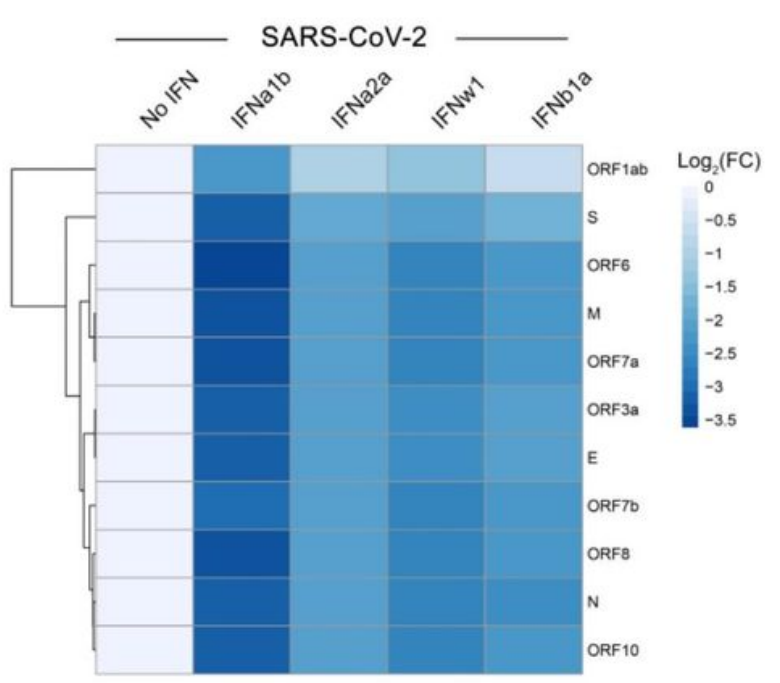

c

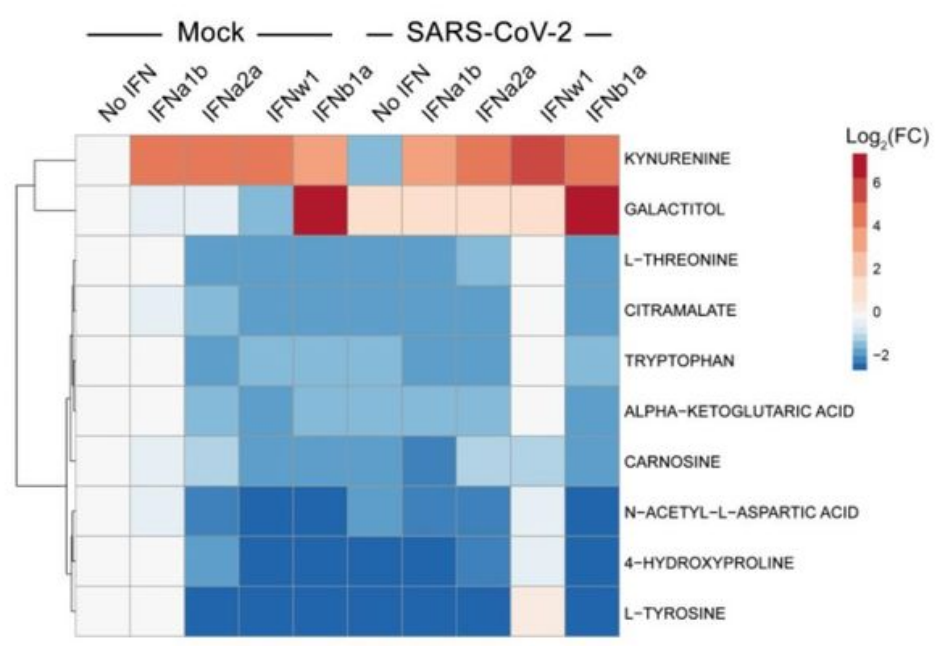

b

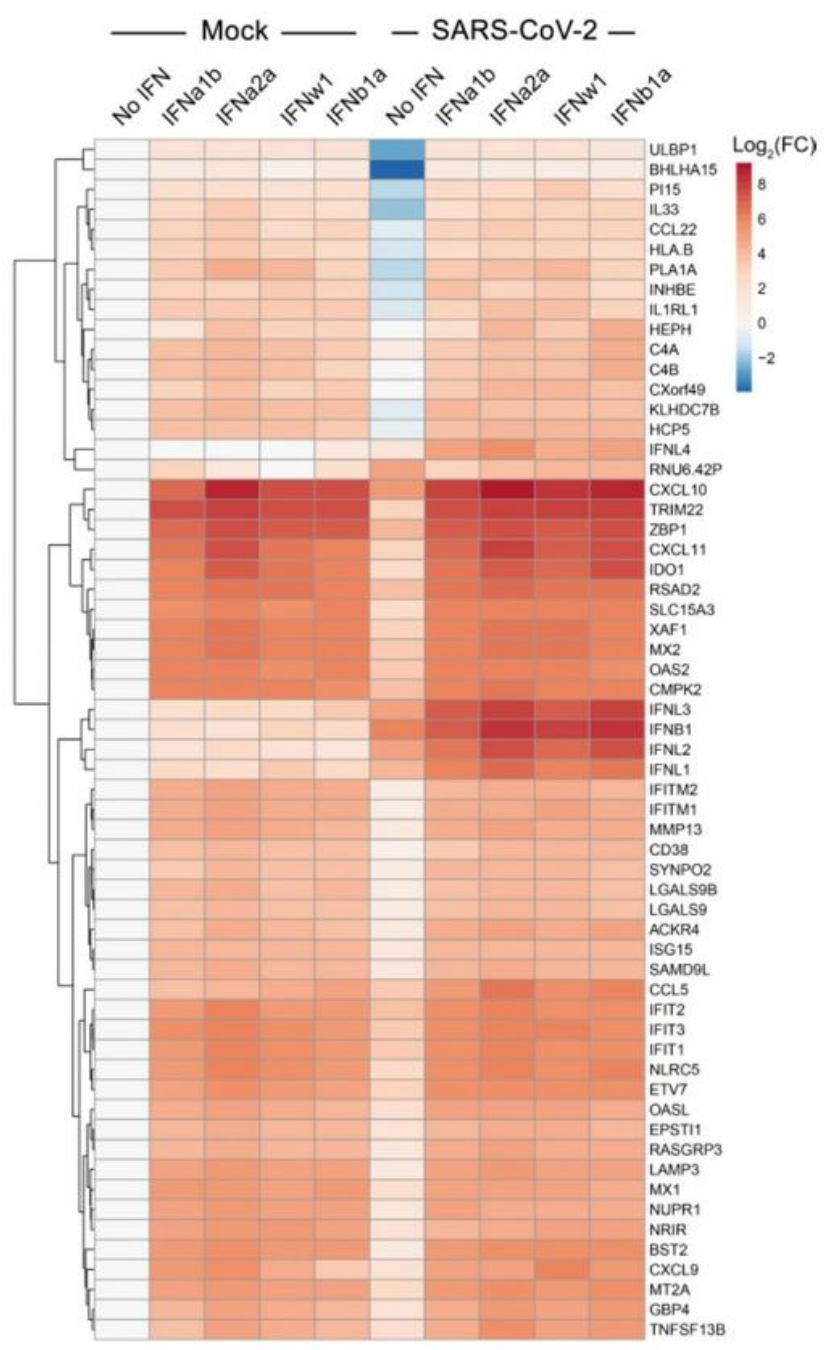

\section{Figure 2}

Transcriptomic and metabolomic analysis of mock- and SARS-CoV-2-infected Calu-3 cells non-treated or treated with type I IFNs. (a) Calu-3 cells were stimulated with IFNs $(1 \mu \mathrm{g} / \mathrm{mL})$ or non-stimulated and infected with SARS-CoV-2 (moi $=0,01)$. A heatmap of viral RNAs affected by treatment is shown. Each cell is colored according to the log2 $\triangle$ transformed expression values of the samples, expressed as fold $\mathbb{Z}$ change relative to the nontreated control. (b) Calu-3 cells were either stimulated with purified recombinant human IFN $(1 \mu \mathrm{g} / \mathrm{mL})$ or left untreated with IFN, then infected with either mock or SARS-CoV-2 (moi = $0,01)$. A heatmap of the most variable cellular genes affected by treatment and virus infection is shown. Each cell is colored according to the log2Хtransformed expression values of the samples, expressed as fold \change relative to the nontreated mockinfected control. (c) Cells were treated as for panel b. After 24 $h$, the cell culture supernatants were collected, and metabolite levels were determined by LC囚MS/MS. A heatmap of the most affected metabolites is shown. Each cell is colored according to the log2】 transferred profiling values of samples, expressed as fold $\llbracket$ change relative to the mock control. 
a

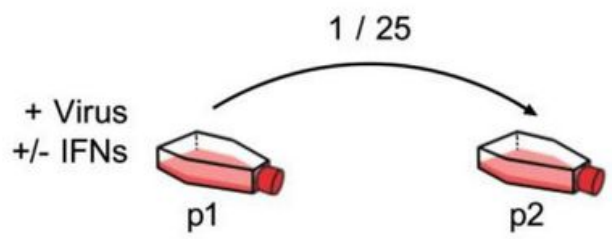

C

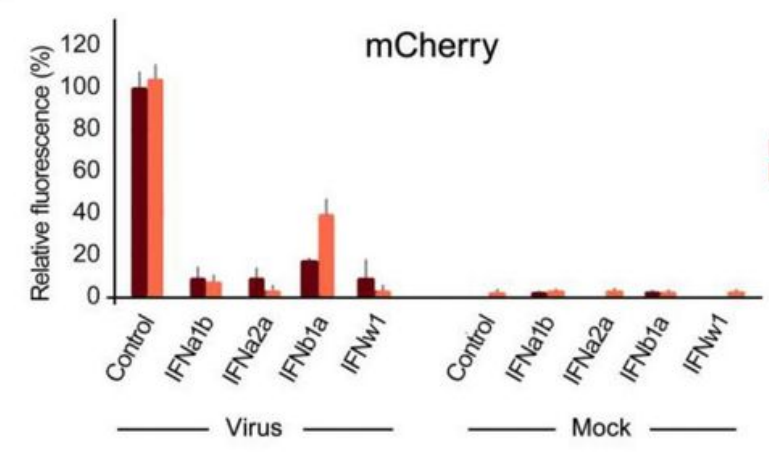

d

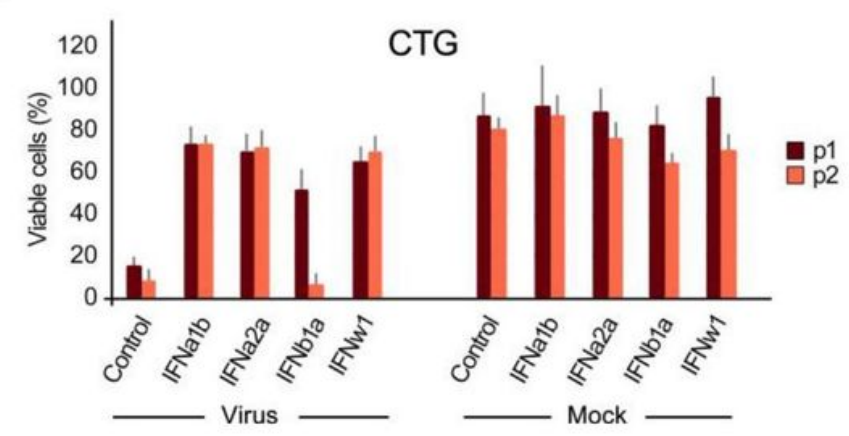

b

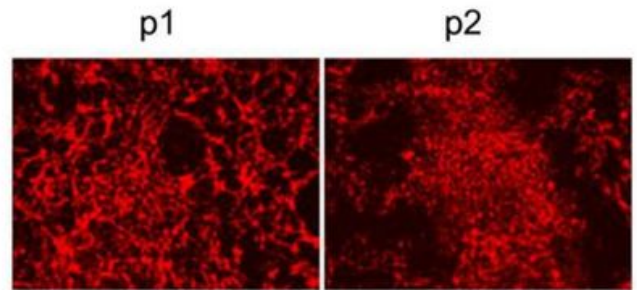

Ctrl
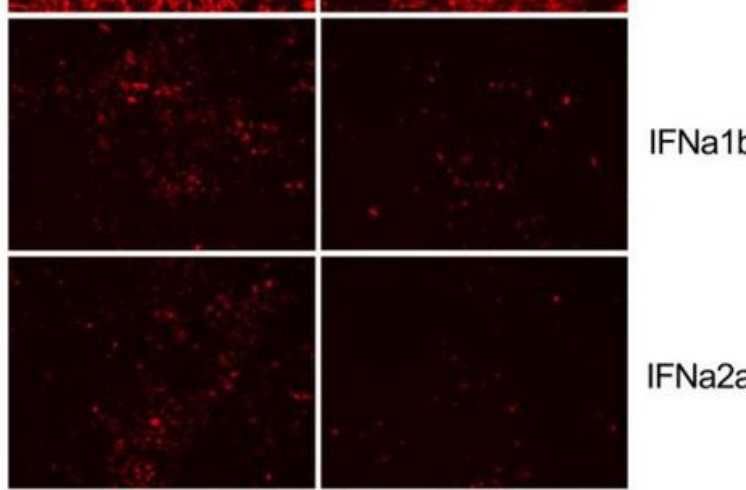

IFNa2a

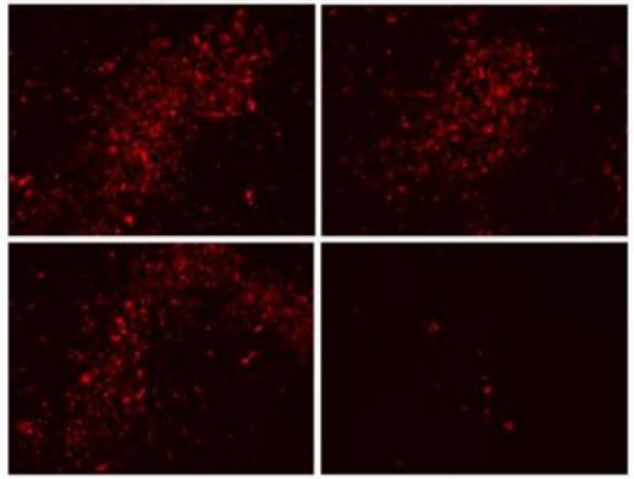

IFNb1a

IFNw1

\section{Figure 3}

IFNa1b, IFNa2a and IFNw1 are more effective than IFNb1a against SARS-CoV-2-mCherry infection in Calu-3 cells. (a) Schematic representation of the experimental setup. (b) Fluorescent images of nontreated (Ctrl) and IFN-treated $(1 \mu \mathrm{g} / \mathrm{mL})$ SARS-CoV-2-mCherry-infected Calu-3 cells (P1) and cells (P2) treated with 25-fold diluted media from P1 cells taken at 48 hpi. (c, d) Fluorescence intensity and viability analysis of P1 and P2 cells performed at 48 hpi. Mock-infected cells were used as controls (Mean \pm SD; $n$ $=3)$.

a

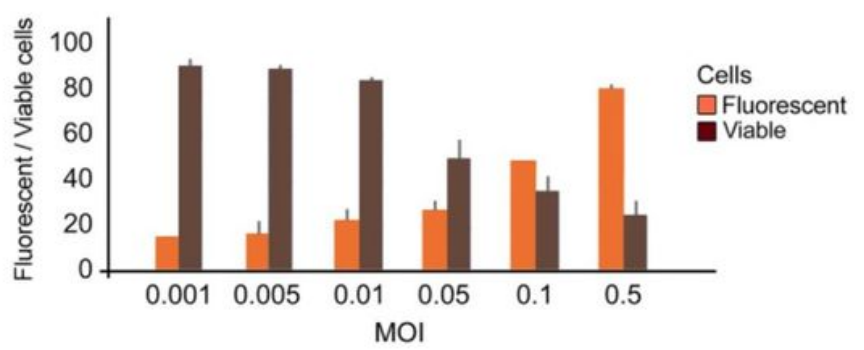

b

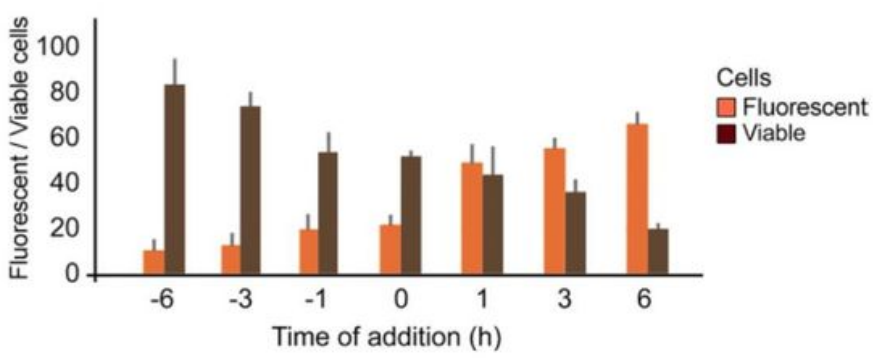




\section{Figure 4}

Anti-SARS-CoV-2 activity of IFNa2a depends on moi and time of administration. (a) Calu-3 cells were treated with $1 \mu \mathrm{g} / \mathrm{mL}$ IFNa2a and infected with indicated moi of SARS-CoV-2-mCherry. Fluorescent intensity and cell viability were measured after $48 \mathrm{~h}$ (Mean $\pm S D ; n=3$ ). (b) Calu-3 cells were treated with $1 \mu \mathrm{g} / \mathrm{mL}$ IFNa2a prior, simultaneously or post infection with SARS-CoV-2-mCherry (moi 0.01). Fluorescent intensity and cell viability were measured after $48 \mathrm{~h}($ Mean $\pm S D ; n=3)$.

a

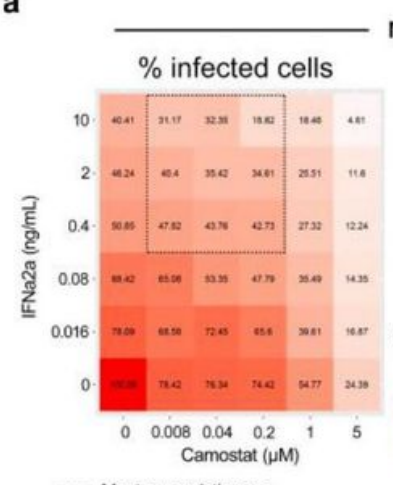

-.-- Most synergistic area
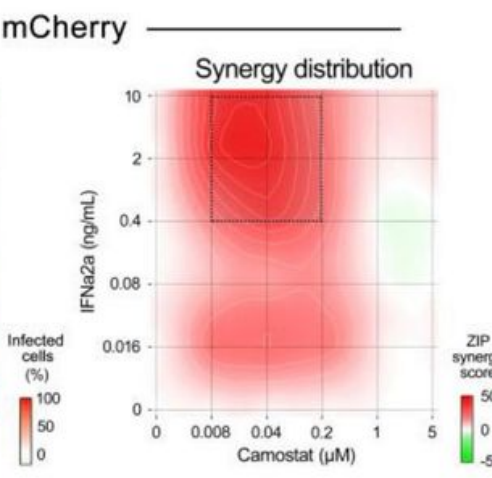

c

\begin{tabular}{|c|c|c|c|}
\hline \multirow{6}{*}{ 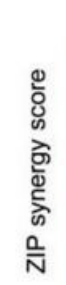 } & IFNa2a plus & mCherry & CTG \\
\hline & Camostat & 14.90 & 23.68 \\
\hline & Conv. serum & 20.99 & 16.98 \\
\hline & EIDD-2801 & 7.94 & 20.07 \\
\hline & Cycloheximide & 5.95 & 25.65 \\
\hline & Remdesivir & 6.38 & 15.75 \\
\hline \multirow{5}{*}{ 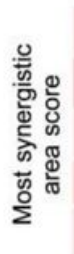 } & Camostat & 28.26 & 33.45 \\
\hline & Conv. serum & 30.57 & 28.16 \\
\hline & EIDD-2801 & 9.16 & 25.75 \\
\hline & Cycloheximide & 12.17 & 38.85 \\
\hline & Remdesivir & 11.69 & 23.55 \\
\hline
\end{tabular}

b
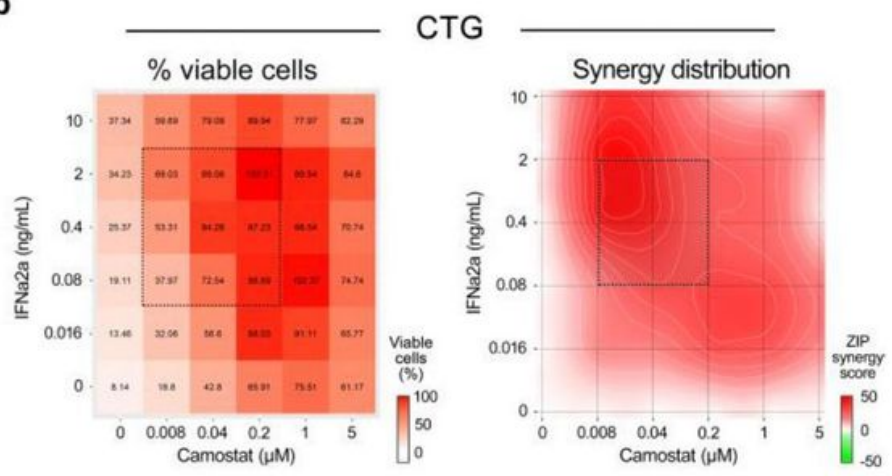

\section{d}

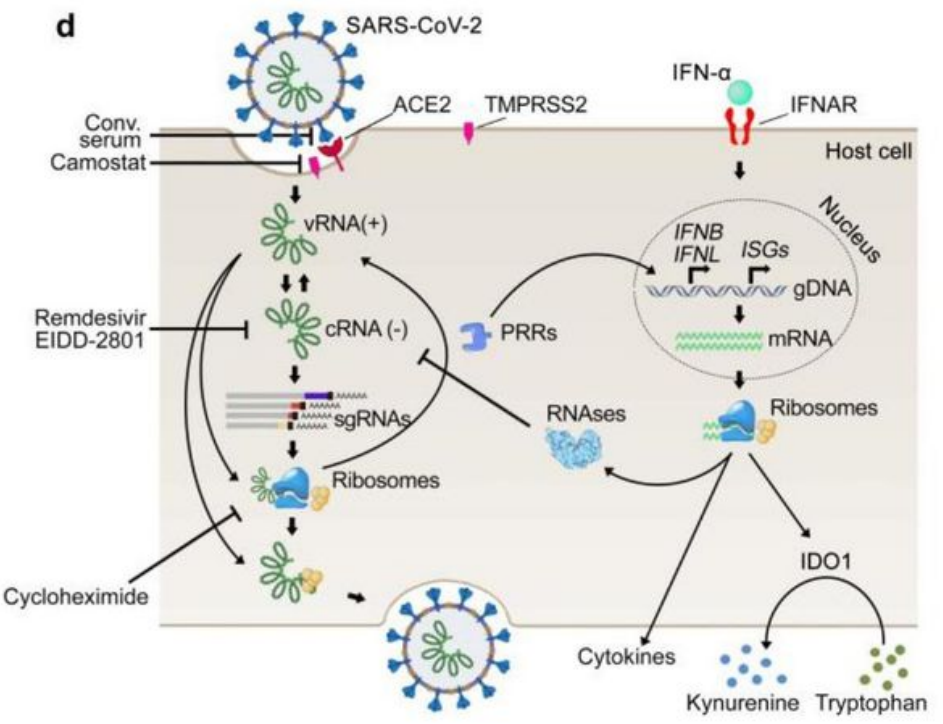

\section{Figure 5}

Synergistic IFNa2a-based combinations against SARS-CoV-2-mCherry infection in Calu-3 cells. (a) The interaction landscape of IFNa2a and camostat in SARS-CoV-2-mCherry infected Calu-3 cells measured using fluorescence (left panel). The interaction landscape of both drugs showing synergy of the drug combination (right panel). (b) The interaction landscapes of IFNa2a and camostat in SARS-CoV-2mCherry infected Calu-3 cells measured using CTG (left panel). The interaction landscape of both drugs showing synergy of the drug combination (right panel). (c) ZIP synergy scores (synergy score for whole $6 \times 6$ dose-response matrices) and the most synergistic area scores (synergy score for most synergistic $3 \times 3$ dose-regions) calculated for indicated drug combinations. (d) Schematic representation of mechanisms of anti-SARS-CoV-2 actions of remdesivir, EIDD-2801, camostat, cycloheximide, and convalescent serum, and stages of virus replication cycle they target. 
a

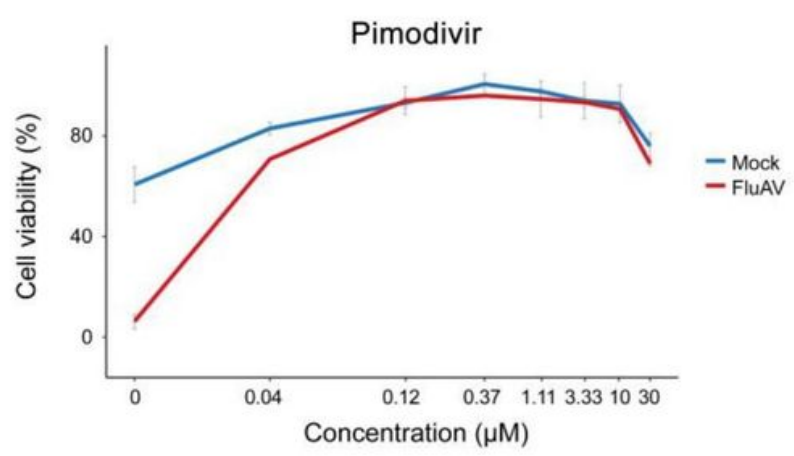

C

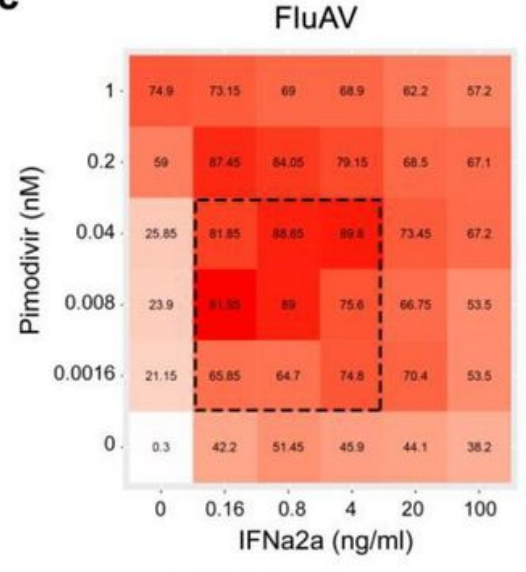

b

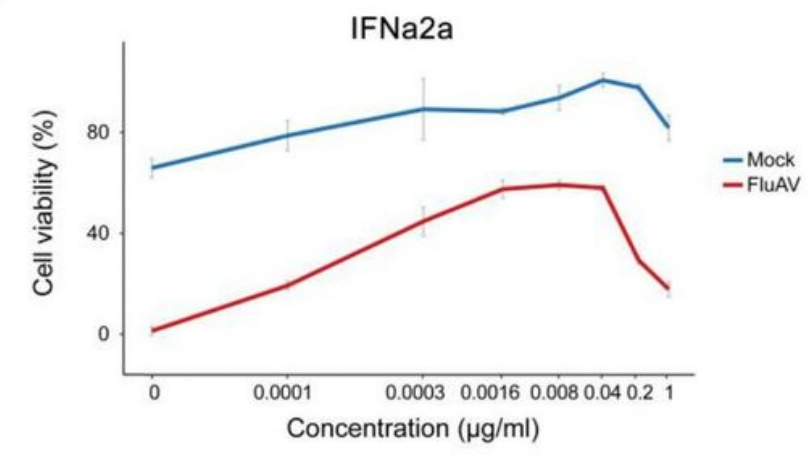

Mock
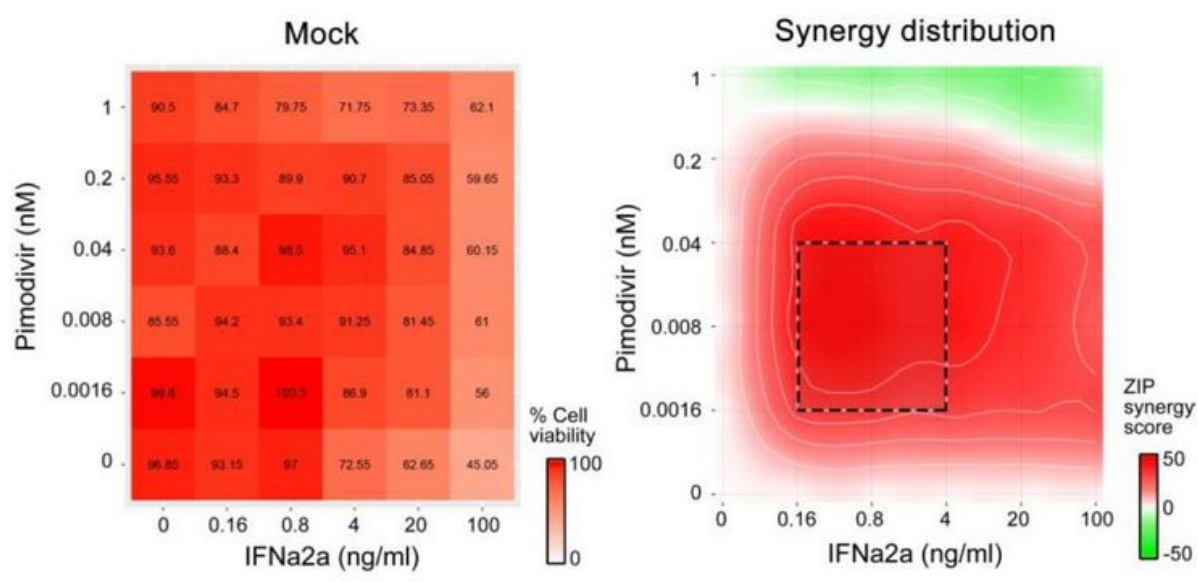

Figure 6

Combination of pimodivir-IFNa2a reduces FluAV infection in A549cells. (a,b) A549 cells were treated with increasing concentrations of pimodivir or IFNa2a and infected with the FluAV (moi $=0.5$ ) or mock. After $48 \mathrm{~h}$, cell viability was determined using a CTG assay. Mean $\pm S D ; n=3$. (c) The interaction landscape of IFNa2a and pimodivir in FluAV- and mock infected A549 cells measured using CTG (left panels). The interaction landscape of both drugs showing synergy of the drug combination (right panel). 
a
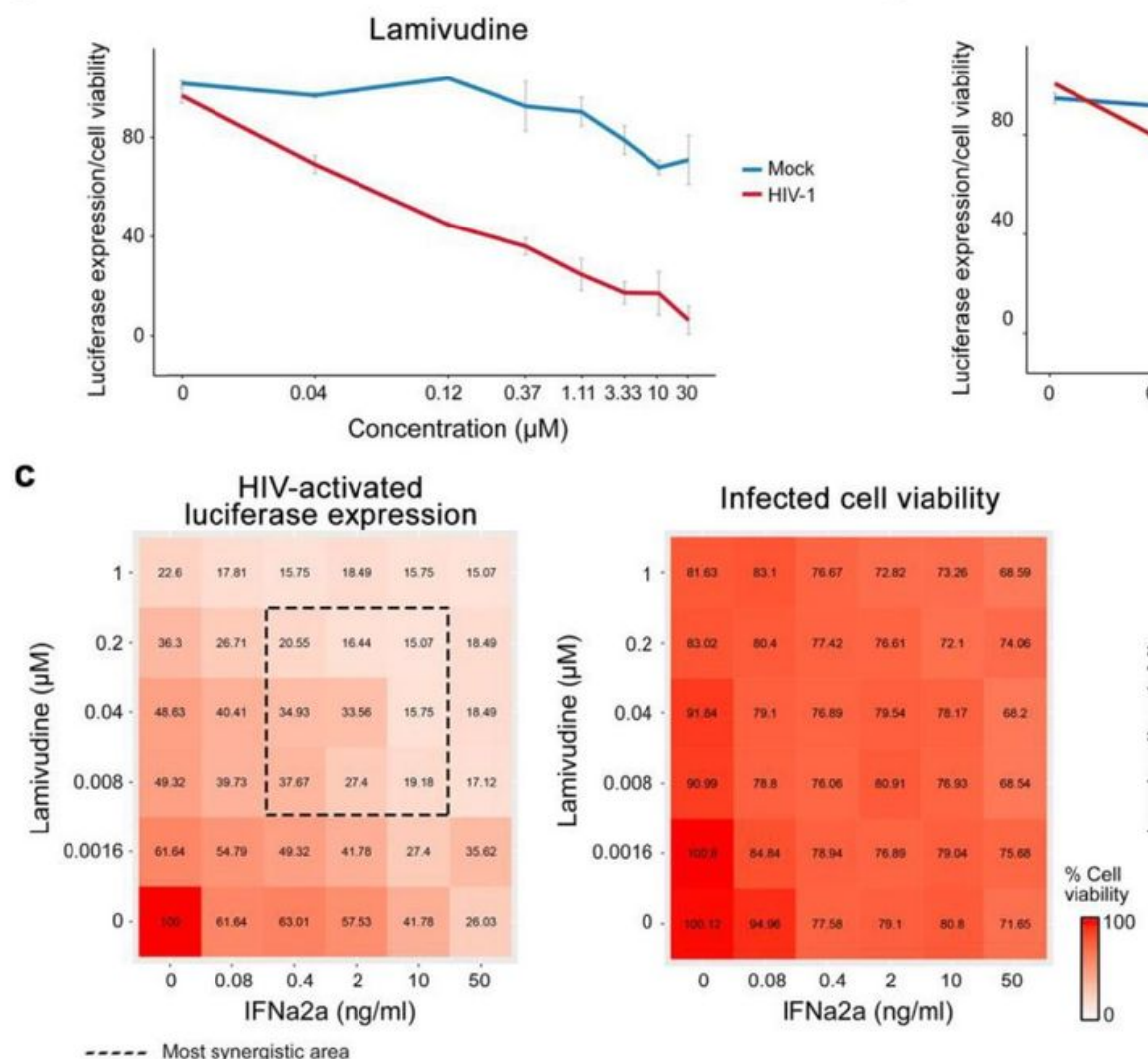

b
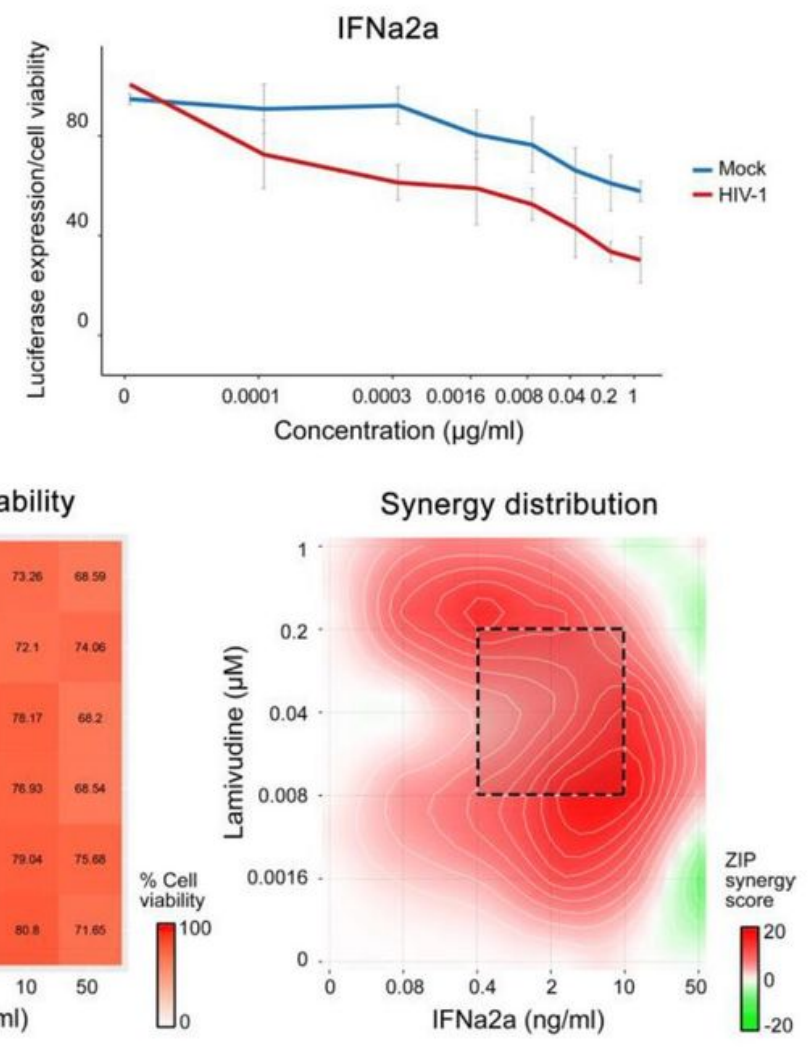

\section{Figure 7}

Combination of lamivudine-IFNa2a reduces HIV-1 infection in TZM-bl cells. $(a, b)$ TZM-bl cells were treated with increasing concentrations of lamivudine or IFNa2a and infected with the HIV-1 or mock. After $48 \mathrm{~h}$, the HIV-activated luciferase expression was measured (red curves). Viability of mock-infected cells was determined using the CTG assay (blue curves). Mean $\pm S D ; n=3$. (c) The interaction landscape of IFNa2a and lamivudine measured using HIV-1-activated luciferase expression in HIV-1- and mockinfected TZM-bl cells, respectively (left panels). The interaction landscape of both drugs showing synergy of their combination (right panel).

\section{Supplementary Files}

This is a list of supplementary files associated with this preprint. Click to download.

- supinfolFN070121.pdf 